\title{
Magnetic Resonance Imaging Study File
}

National Cancer Institute

\section{Source}

National Cancer Institute. Magnetic Resonance Imaging Study File. NCI Thesaurus. Code C115501.

A collection of magnetic resonance image files pertaining to a clinical study. 